\title{
Surface Organization of Orientation and Direction Selectivity in Cat Area 18
}

\author{
N. V. Swindale, ${ }^{1}$ J. A. Matsubara, ${ }^{1,3}$ and M. S. Cynader ${ }^{1,2}$ \\ Departments of ${ }^{1}$ Psychology, ${ }^{2}$ Physiology, and ${ }^{3}$ Anatomy, Dalhousie University, Halifax, B3H 4J1, Canada
}

Two-dimensional maps of orientation and direction preference were made in area 18 of the cat's visual cortex using multiple electrode penetrations 150-300 $\mu \mathrm{m}$ apart. The maps were then analyzed by autocorrelation and Fourier transformation. The power spectrum of the orientation map was sharply peaked below the theoretical cutoff frequency for the sampling function (the pattern of electrode penetrations) used to obtain the map. This suggested that it would be possible to interpolate orientation values between the sample points in the cortex. This was done and the resulting finegrain maps of orientation preference were studied. Empirical testing showed that the interpolated orientation values were accurate to within $\pm 30^{\circ}$. Study of the fine-grain maps showed that iso-orientation domains for broad orientation ranges $\left(0^{\circ}-\right.$ $\left.9^{\circ}\right)$ were branching bands running from an anterolateral to a posteromedial direction across the surface of the cortex with a periodicity of $1.25 \pm 0.13 \mathrm{~mm}$. Domains for smaller orientation ranges $\left(0^{\circ}-30^{\circ}\right)$ were periodically spaced, but somewhat irregularly shaped patches. The orientation maps contained numerous point singularities where orientation changed discontinuously. These were spaced about $750 \mu \mathrm{m}$ apart and most were surrounded by a single $180^{\circ}$ cycle of orientations. Autocorrelation analysis of the maps of preferred direction revealed local clustering that extended over a distance of 250-300 $\mu \mathrm{m}$, but spectral analysis failed to reveal any evidence of periodicity. The absence of periodicity was probably due to a relatively large number of $180^{\circ}$ reversals in the map of direction preference, which do not affect the continuity of the orientation map and are not sufficiently numerous to destroy local continuity in the direction map. It is shown that the direction map, if it is to be as continuous as possible, must nevertheless contain lines across which direction preference reverses by $180^{\circ}$. These lines run from one orientation singularity to another. Evidence that the direction map approaches this degree of continuity is presented.

After discovering that neurons in the visual cortex of cats and monkeys were sensitive to the orientation of a bar or an edge stimulus, Hubcl and Wiesel $(1962,1963,1968)$ went on to show how preferred orientation varied with position in the cortex. In

\footnotetext{
Received June 10, 1986; revised Sept. 15, 1986; accepted Oct. 2, 1986.

We thank Julia Hickey, Lori Colpitts, Gordon Troop, and David Conrad for technical assistance. This work was supported by grants from the MRC (PG-29) and NSERC (A9939) of Canada to M.C.

Correspondence should be addressed to N. V. Swindale, Dept. of Psychology, Dalhousie University, Halifax, B3H 4Jl, Nova Scotia, Canada.

Copyright (C) 1987 Society for Neuroscience $0270-6474 / 87 / 051414-14 \$ 02.00 / 0$
}

the cat they found that cells in the same vertical column of tissue had the same orientation preference, while cells in adjacent columns had preferences that differed slightly. A similar but more detailed study of monkey area 17 (Hubel and Wiesel, 1974) showed that over distances of 1-2 $\mathrm{mm}$, preferred orientation changed linearly as a function of distance through the cortex, a single $180^{\circ}$ cycle of change taking about $500-600 \mu \mathrm{m}$ of lateral movement to complete. Linear orientation sequences were often unpredictably interrupted by reversals in the direction of change (i.e., from clockwise to counterclockwise rotation) or by discontinuous jumps in orientation preference up to $90^{\circ}$ in size. Although somewhat less orderly, the same arrangement seems to prevail in area 17 of the cat (Hubel and Wiesel, 1963, 1974; Albus, 1975, 1985) and in other primates (Humphrey and Norton, 1980).

Although the properties of the orientation map, as seen in 1 dimension by a recording electrode moving laterally through the cortex, are well established, the nature of the 2-dimensional layout of orientations has remained uncertain, owing to the difficulty of recording the preferred orientation from many points spread sufficiently densely over a large area of the cortex. Activity labeling with 2-deoxyglucose (Hubel et al., 1978; Albus, 1979; Humphrey et al., 1980; Singer, 1981; Albus and Sieber, 1984) has shown that iso-orientation domains for relatively broad orientation ranges $\left(45^{\circ}-60^{\circ}\right.$ wide) are periodically spaced and, in the cat and tree shrew, stripe-like in their morphology. The 2-deoxyglucose technique has a relatively coarse resolution, however, and has not shown how the domains for narrow orientation ranges are arranged relative to one another.

On the basis of their 1-dimensional maps, obtained from single-electrode penetrations, Hubel and Wiesel (1974) and, more recently, Livingstone and Hubel (1984) have argued that isoorientation domains are likely to be uniformly thin parallel slabs of tissue, perhaps oriented at right angles to ocular dominance bands. In contrast, Braitenberg and Braitenberg (1979) proposed that orientations might be arranged radially, like the spokes of a wheel, around periodically spaced organizing "centers," about $300 \mu \mathrm{m}$ apart. Swindale $(1982,1985)$ suggested a similar arrangement in some respects, in which orientations are arranged continuously around $180^{\circ}$ singularities that have a spacing of about half that of the iso-orientation domains. Recently, Blasdel and Salama (1986) used voltage-sensitive dyes to map the layout of orientation preference in monkey visual cortex, and their results appear to confirm the latter suggestion: color-coded maps of orientation preference show numerous $180^{\circ}$ singularities (points where a single complete set of color domains meet) with a spacing of about $200-300 \mu \mathrm{m}$.

There have been few studies of the spatial layout of direction 
preference in the visual cortex. Payne et al. (1981) and Tolhurst et al. (1981) have provided evidence from 1-dimensional maps in area 17 of frequent $180^{\circ}$ reversals in direction preference, as well as of a local clustering extending over lateral distances of up to about $300 \mu \mathrm{m}$. Tolhurst et al. (1981) also present evidence that direction preference, like orientation preference, remains constant with depth in the cortex, i.e., has a columnar organization. How direction preference might be overlaid on the map of orientation preference is still unclear, however.

We studicd the layouts of both orientation and direction preference in the cat's visual cortex by recording from a large number of penetrations spaced about $150-300 \mu \mathrm{m}$ apart in a quasiregular grid across an exposed flat surface of area 18 . The maps were analyzed by means of autocorrelation and Fourier analysis. $\mathrm{A}$ method for reconstructing a fine-grain orientation map from the initial relatively coarse physiological one is presented, and it is shown that these fine-grain maps contain numerous $180^{\circ} \mathrm{sin}$ gularities. We suggest that direction selectivity may be overlaid on the map of orientation preference in a way that maximizes its continuity, although, given the presence of singularities in the orientation map, it is inevitable that there will be lines of $180^{\circ}$ discontinuity in the direction map. These lines begin and end in singularities.

These studies form part of a series of experiments in which we examined orientation and direction selectivity and its relation to other physiological and anatomical properties in area 18 of the cat's visual cortex. Our findings are described in accompanying papers (Cynader et al., 1987; Matsubara et al., 1987).

\section{Materials and Methods}

Recordings were made from anesthetized and paralyzed cats (see $\mathrm{Cy}$ nader et al., 1987, for further details) held in a stereotaxic apparatus. A large craniotomy was made just lateral to the midline, and the dura removed. The surface pattern of blood vessels was photographed, together with a scale bar, and served as a reference for establishing the position of electrode penetrations. A sealed Davies chamber containing a transparent Plexiglas plate was cemented to the skull around the craniotomy. This substantially reduced pulsations of the brain and increased recording stability. The region of arca 18 that was studied, about $1-4$ $\mathrm{mm}$ lateral to the midline and $0-5 \mathrm{~mm}$ anterior to ear bar 0 , is relatively flat, so that the position marked on the photograph was a good approximation to the position in tangential cortical coordinates.

To minimize damage to the cortex, penetrations were made with very fine glass-coated platinum-iridium microelectrodes with a shaft diameter $<80 \mu \mathrm{m}$ at the level of entry into the cortex. Penetrations were normally spaced $200-300 \mu \mathrm{m}$ apart, although occasionally there were pairs closer together than this, and in some maps the average spacing was made deliberately less. Before entering the cortex, the electrode was positioned so as to avoid damage to blood vessels. Recordings were made in the superficial layers of the cortex at a depth of around 400$700 \mu \mathrm{m}$ below the surface, which minimized distortion of the map due to curvature of the cortex. Damage caused by the penetrations was sufficiently limited that subsequent histology normally failed to reveal the positions of the penetrations.

The preferred orientation and direction were determined by judging the best single- or multiunit response to a moving bar or edge stimulus, as heard on an audiomonitor. The accuracy of the orientation measurements was probably about $\pm 15^{\circ}$. About $80-150$ penetrations were made in a typical experiment, taking 1-2 d to complete. Subsequently, the position of each penetration, as recorded on the photograph, and the preferred orientation and direction at that point (or values signifying nondirectional or nonoriented responses) were stored on disc in a computer. Data from a total of 24 animals were available; 16 of the most complete and extensive maps of orientation and direction selectivity were used for the analyses described here.

Data analysis. The representation of orientation values in a manner allowing numerical analysis, such as Fouricr transformation, is not straightforward. Using the normal angular measure (i.e., numbers from
0 to 360 ) is unsatisfactory for 2 reasons: First, values that differ numerically by a large quantity (e.g., 179) can have similar orientations (showing that modular arithmetic is not a solution to the problem); second, orientation values cannot be averaged with sensible results (e.g., the average of a uniform distribution of orientations over the range $0^{\circ}-$ $360^{\circ}$ would be $180^{\circ}$ ). The natural solution is to use a vector representation, i.e., to have the vector $\mathbf{z}$ with components $a=\cos (\theta), b=\sin (\theta)$, but this introduces a further problem: since orientation values differing by $180^{\circ}$ give rise to different values of $\mathbf{z}$, the correspondence between measured orientation and the value of $z$ is not unique. Also, orientations (as opposed to directions) still cannot be averaged with sensible results, e.g., the average of a uniform distribution over the range $0^{\circ}-180^{\circ}$ is the vector $(0,1)=90^{\circ}$. The solution to the problem that was adopted here, as elsewhere (Batschelet, 1981; Swindale, 1982, 1985), was to let $a=$ $\cos (2 \theta)$ and $b=\sin (2 \theta)$, i.e., to double the orientation angle before resolving it into components. There is then a unique correspondence between the values of $\mathbf{z}$ and experimentally measured orientation; smoothly changing orientations give rise to smoothly changing values of components $a$ and $b$, and the average of a uniform distribution of orientations over a $180^{\circ}$ range is the vector $(0,0)$.

Note that when $b=0$, the orientation $\theta=0.5 \tan ^{-1}(b / a)$ is either $0^{\circ}$ (when $a$ is positive) or $90^{\circ}$ (when $a$ is negative). When $a=0$, the orientations represented are similarly either $45^{\circ}$ or $135^{\circ}$, depending upon the sign of $b$.

Since direction preference is a quantity that is cyclic over a $360^{\circ}$ range, its appropriate representation is the simpler one, where the components of the vector $\mathrm{z}$ are $a=\cos (\theta)$ and $b=\sin (\theta)$.

Fourier analysis of the data was performed using a 2-dimensional fast Fourier transform (Brigham, 1974) of floating point data stored in a $64 \times 64$-point array. The real and imaginary parts of the data were the components $a$ and $b$, determined as described above, with unitary modulus. Nonoriented points and, when data on direction selectivity were being analyzed, non-direction-selective points, had the value $(0,0)$, i.e. they were omitted from the data. Positional values were first determined from the photograph showing the blood vessel pattern and the points of entry of each penetration, and were then scaled to fit into the array. Because the fast Fourier transform treats opposite edges of an array as though they are in continuity (i.e., as though this is a unit cell in an infinitely repeating 2-dimensional pattern), it is incorrect to scale the positional values of the data points so that the whole of the array is occupied (see Bergland, 1969)-the difference in orientation values of points at opposite ends of the map will give rise to spurious discontinuities in the data. To minimize this problem, the position values were normally scaled so that the longest side of the map occupied between 0.5 and 0.75 ( 32 and 48 units) of the linear dimensions of the array. The accuracy with which position on the cortex could be represented in this way was about $70-100 \mu \mathrm{m}$. Since the orientation changes, on average, by about $180^{\circ}$ in $1.2 \mathrm{~mm}$ (see Results), this positional inaccuracy corresponds to an inaccuracy in the measure of orientation of about $13^{\circ}$. This is about equal to the inaccuracy of the experimental measurements. Programs for analysis were written in Fortran and run on a PDP $11 / 34$ computer. Program logic was tested by substituting for the data either randomly chosen orientations, or orientations that changed sinusoidally as a function of one direction across the array.

\section{Results}

\section{The autocorrelation function}

Figure 1 shows the cumulative 2-dimensional autocorrelation function for the orientation data in 21 maps of area 18. The figure shows, in vector form, the average difference in orientation preferences of all pairs of points in the maps as a function of their anteroposterior (A-P) and mediolateral (M-I.) separation. The 2 axes of the diagram are thus in cortical distance coordinates: The horizontal axis to the right of the origin of the graph corresponds to relative separation in a medial direction, and that to the left of the origin corresponds to relative separation in a lateral direction. The vertical axis corresponds to relative separation along the A-P axis. Average orientation was computed by adding the orientation vectors of the orientation differences between each pair of points for each separation. The 
Figure 1. The 2-dimensional autocorrelation function for 21 maps of orientation preference in area 18. Shown is the average difference in preferred orientation between pairs of points as a function of their $M-L$ (mediolateral) and $A-P$ (anteroposterior) separation The origin is marked at the center of the graph and points are scaled to be $100 \mu \mathrm{m}$ apart. Note that the upper half of the figure is symmetrically related to the bottom half. The reason for this is explained in the text. The direction of the axes of the figure is appropriate for the right hemisphere: Since some of the maps came from the left hemisphere, the direction of their mediolateral axes was reversed so that they would correspond to those from the other side. Circles mark points illustrated in Figure 2 .

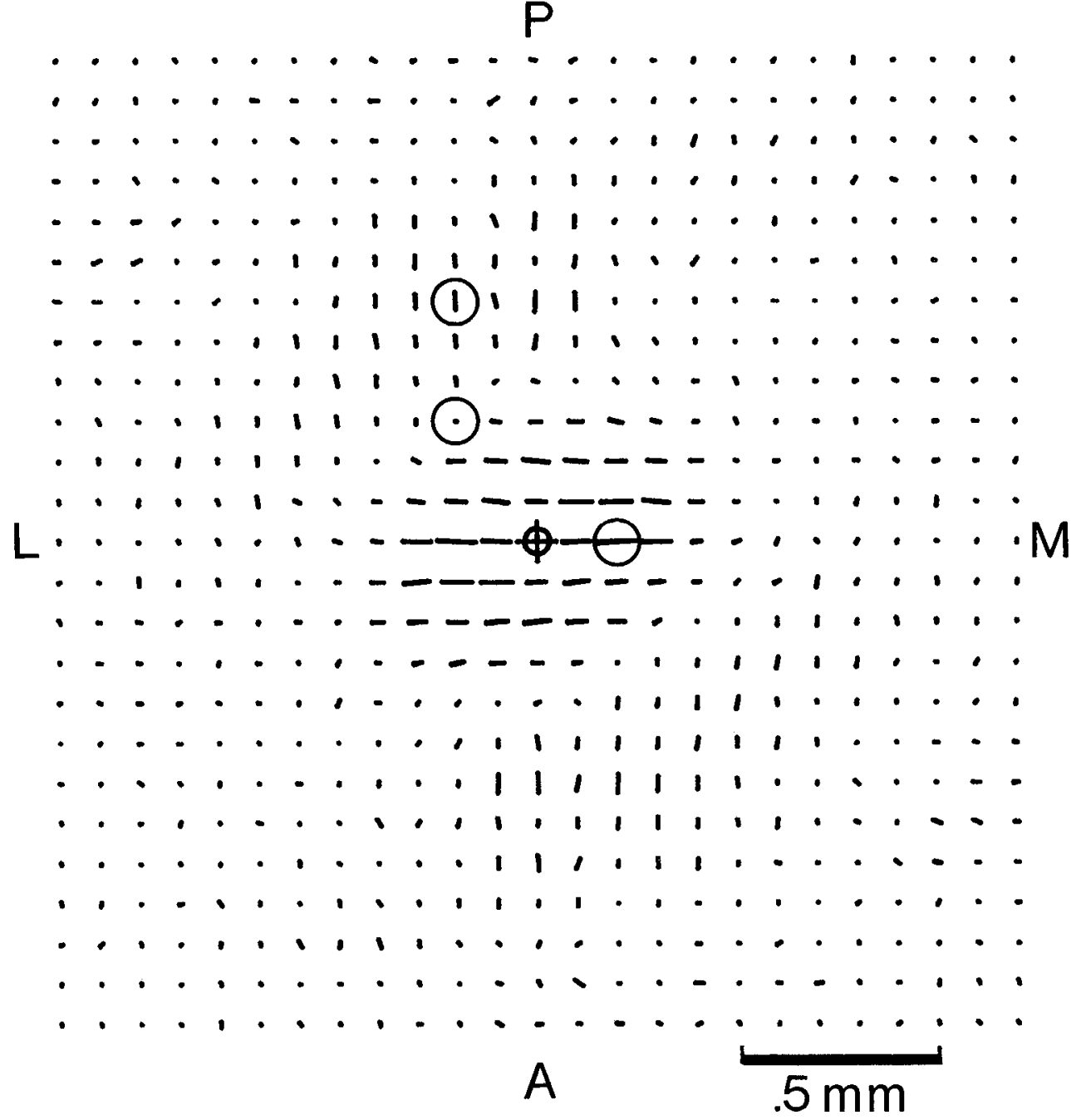

of elongation, and at a distance about half the period of the domains from the origin, where orientations differed on average by $90^{\circ}$. The fact that average orientation does not change in any consistent clockwise or anticlockwise way as one moves out from the origin in Figure 1 implies that orientation changes clockwise as often as anticlockwise when moving in a particular direction across the cortical surface.

Each of the vectors in Figure 1 is the average of an underlying distribution of orientation differences. Since different distributions can have the same average, it is informative to examine the underlying distribution; Figure 2 shows this for 3 of the points in Figure 1: (1) a region $200 \mu \mathrm{m}$ from the origin, (2) a region about $350 \mu \mathrm{m}$ distant from the origin, within which orientation preferences are uncorrelated, and (3) a region about $650 \mu \mathrm{m}$ distant, within which orientations differ on average by $90^{\circ}$ from that at the origin.

A similar set of analyses was performed on the maps of direction selectivity, omitting points that showed no overall direction preference, and using a straightforward vector representation to obtain the average differences in the preferred directions of points a given distance apart. The resulting 2-dimensional autocorrelation map (Fig. 3) shows a tendency for points in area 18 less than $250 \mu \mathrm{m}$ apart to have the same preferred direction. For separations greater than this the correlations are weak and inconsistent. Examination of the actual distribution of direction this direction, and a region in a direction orthogonal to this axis 

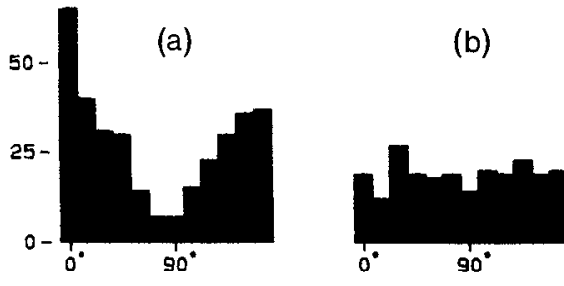

Relative Orientation (c)

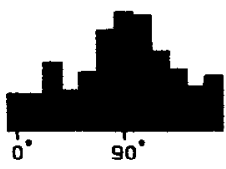

Figure 2. Histograms of the distribution of orientation differences underlying the averaged vectors for 3 of the points in Figure 1 (marked by circles). $a$, From the point with coordinates $(2,0)$ relative to the origin in Figure 1, i.e., $200 \mu \mathrm{m}$ to the right of it, within which orientation preferences tend, on average, to be the same. $b$, From a point about $350 \mu \mathrm{m}$ from the origin (coordinates $-2,3$ ) within which orientation preferences is uncorrelated. $c$, From a region, approximately $650 \mu \mathrm{m}$ from the origin (coordinates $-2,6$ ), within which orientation preferences differ on average by $90^{\circ}$.

differences underlying the local correlation in preferred direction (Fig. $4 a$ ) shows 2 peaks, one at $0^{\circ}$, implying a similar preferred direction, and a second, smaller peak at $180^{\circ}$, implying the same orientation preference, but opposite preferred directions. The size of this smaller peak grows with distance (Fig. $4 b$ ); at distances greater than $350 \mu \mathrm{m}$ the distribution is effectively flat
(Fig. $4 c$; the slight peak at $180^{\circ}$ is not consistently present in other graphs from neighboring points at similar distances from the origin). In regions where orientation preferences tend to be orthogonal, there are 2 peaks of similar size at $90^{\circ}$ and $270^{\circ}$ (not shown).

\section{Power spectrum}

Figures 5 and 6 show power spectra, obtained form the Fourier transforms of the orientation and direction data from 4 animals $(a-d)$. The spectra can be interpreted as follows:

1. The energy at zero frequency is a measure of the average orientation, or dircction, of the data values. Thus, if all orientations, or nearly all, are represented in the data, there will be little energy present at zero frequency. This was the case for many of the animals studied (e.g., Fig. 5, $a, c, d$ ), though in others (e.g., Fig. $5 b$ ) energy was present in variable amounts, perhaps because the number of measurements in the sample was not sufficient to ensure a uniform average. Even where the average is close to zero, it is not necessarily the case that the orientation distribution is uniform over a $180^{\circ}$ range, since an excess of one orientation value can be counterbalanced by a similar excess at an orthogonal orientation. Direct examination of the orientation distribution (Fig. 7, left) shows that horizontal and vertical orientations do predominate to some extent, as has been reported by other investigators for area 17 of the cat (e.g.,

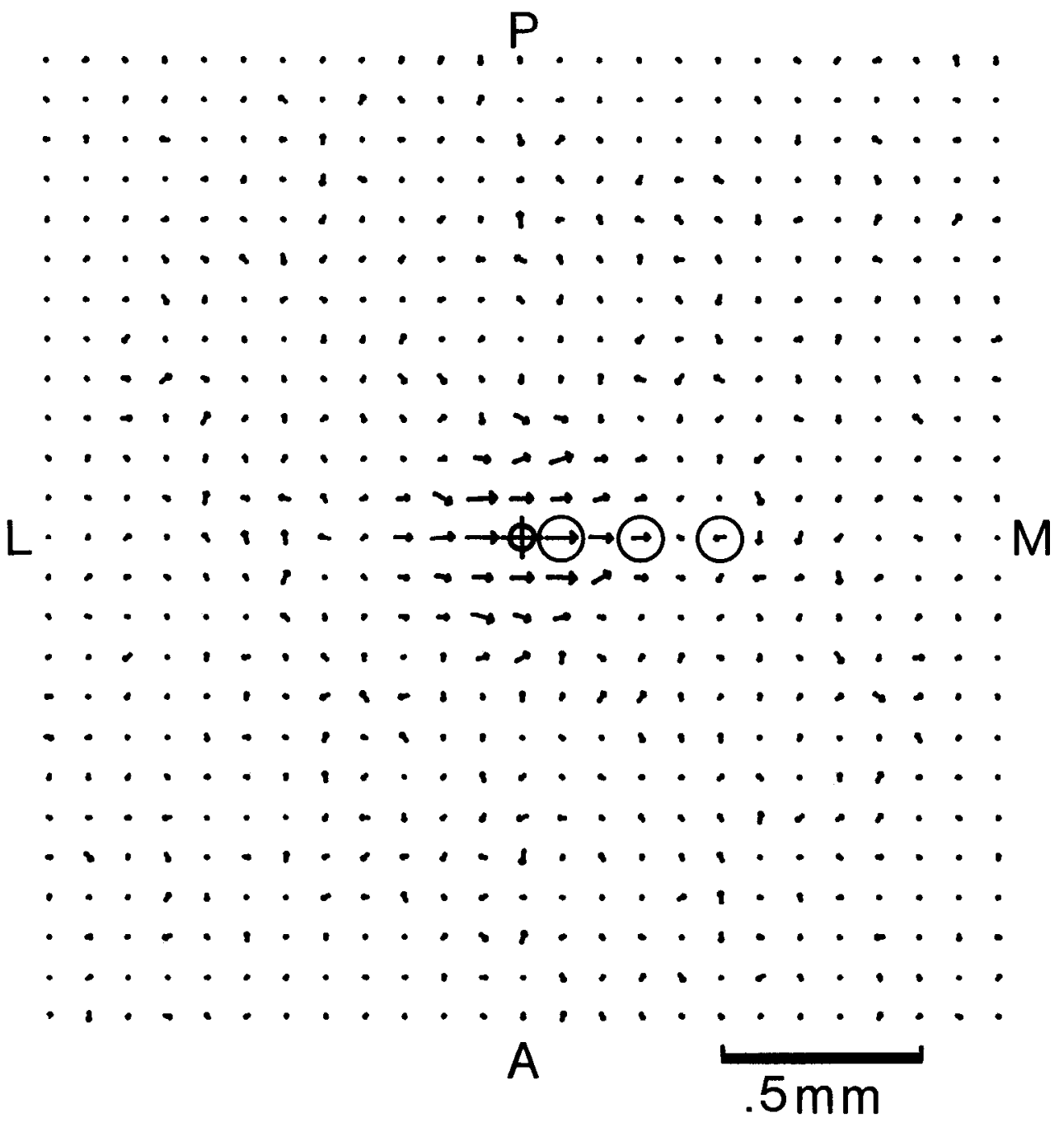

Figure 3. Two-dimensional autocorrelation function for 21 maps of direction preference in area 18 , obtained in a manner similar to that shown in Figure 1. Points in the maps less than about $300 \mu \mathrm{m}$ apart tend, on average, to have similar direction preferences. The direction preferences of points further apart than this are not consistently correlated. Circles mark points illustrated in Figure 4. 


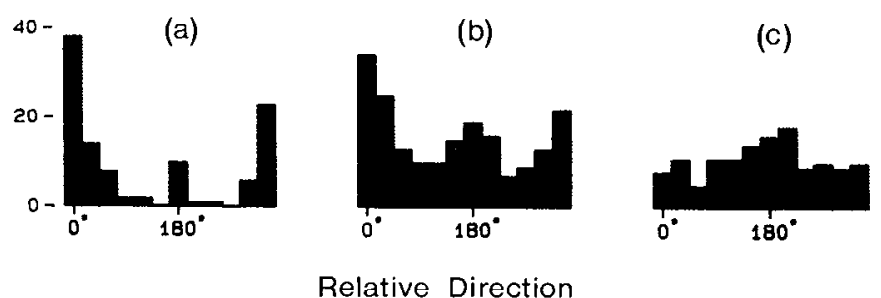

Figure 4. Histograms of the distribution of differences in direction preference for 3 of the points (marked by circles) in Figure 3. a, From the point with coordinates $(1,0)$, i.e., $100 \mu \mathrm{m}$ to the right of the origin of Figure 3. $b$, From the point with coordinates $(3,0)$. $c$, From the point with coordinates $(5,0)$.

Leventhal and Hirsch, 1977; Kennedy and Orban, 1979). A similar argument can be applied to the direction data (Fig. 7, right) which show a slight preponderance of cells exhibiting a preference for downward directions of movement.

2. The orientation power spectrum is sharply peaked at a particular distance from the origin (i.e., the leftmost bin in each of the histograms of Figs. 5 and 6), the energy usually being concentrated over a narrow range of directions relative to the origin. The presence of a peak implies that orientation tends to change smoothly and periodically with distance across the cortical surface. The distance of the peak from the origin is inversely proportional to the overall periodicity of the orientation map (i.e., the further the peak from the origin, the higher the spatial frequency and the smaller the spacing of the iso-orientation domains). Because different scaling factors were used in different animals, the position of the peak in the histogram varied; however, calculation of the actual periodicity of the domains in millimeters gave similar values, averaging $1.25 \pm 0.13 \mathrm{~mm}$ in 16 animals. The fact that the peak occupies a narrow range of directions relative to the origin implies that the iso-orientation domains have an overall direction of elongation. This direction is orthogonal to the angle between the peak in the power spectrum and the origin; this was most commonly in a direction about $30^{\circ}$ anterior relative to a $\mathrm{M}-\mathrm{L}$ axis. In some animals, however, there was more than 1 peak (e.g., Fig. $5 a$ ), and in one animal the energy was spread in a nearly complete annulus around the origin.

Beyond the peak in the spectrum, and above the theoretical sampling limit (see Appendix), there was a roughly uniform low level of energy at all frequencies and directions, up to the maximum frequency represented in the discrete array used for transformation. We show in the Appendix that (1) at least some of

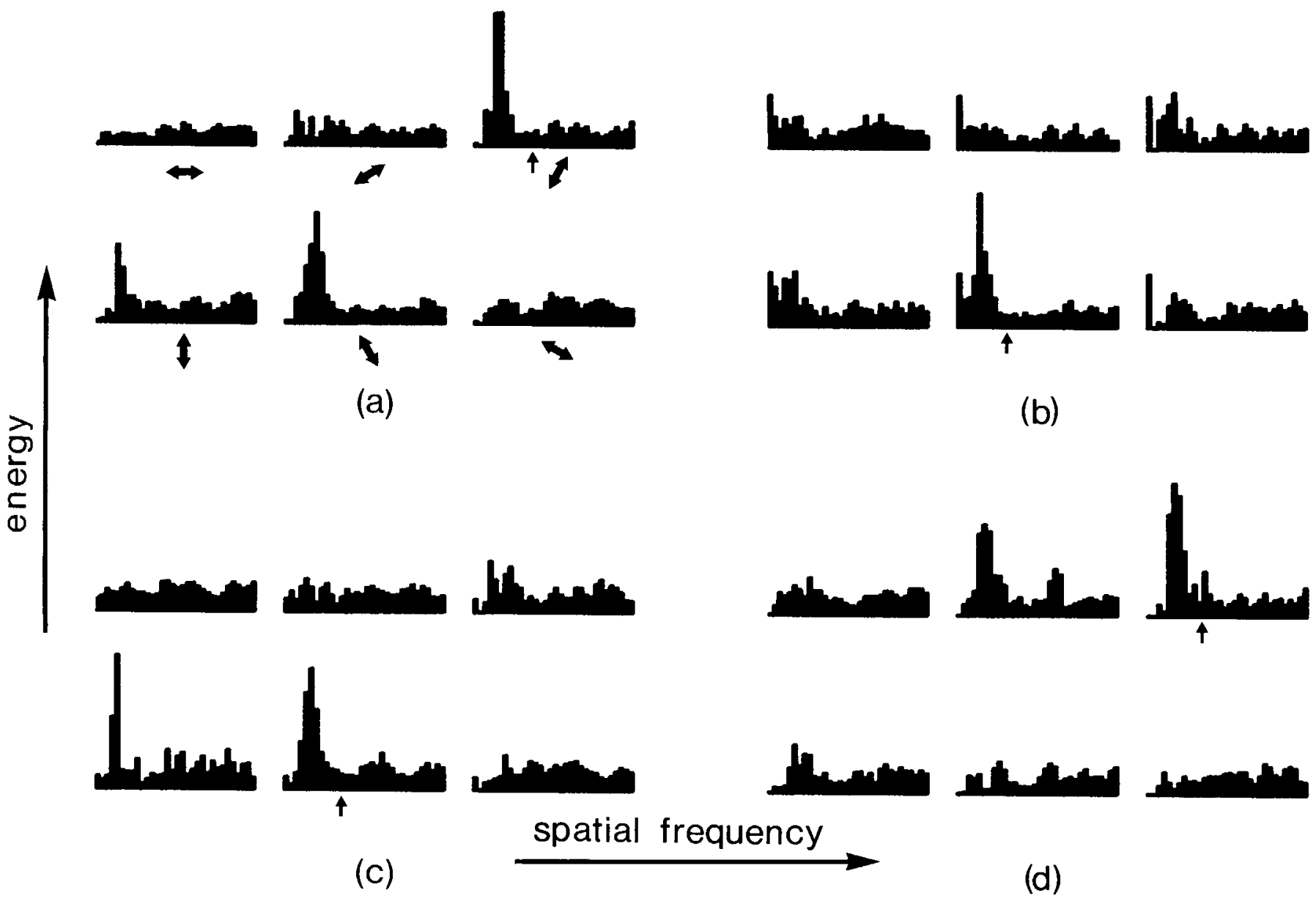

Figure 5. $a-d$, Fourier power spectra from the orientation maps of 4 different animals. Six histograms are shown for each animal. Each shows energy per unit area as a function of radial distance along each of 6 different sectors in 2-dimensional frequency space. Each sector is $30^{\circ}$ wide, and, in reading order, are arranged in the sequence $0^{\circ}-30^{\circ}, 30^{\circ}-60^{\circ}$, etc., as indicated by the double-headed arrows beneath the first set of histograms in $a$. Note that the peaks occur only in some of the histograms, indicating that energy is concentrated not only at a particular distance from the origin, but within a narrow range of directions relative to it. The small, single-headed arrows indicate the theoretical cutoff frequency for the particular electrode spacing used to obtain the map. 


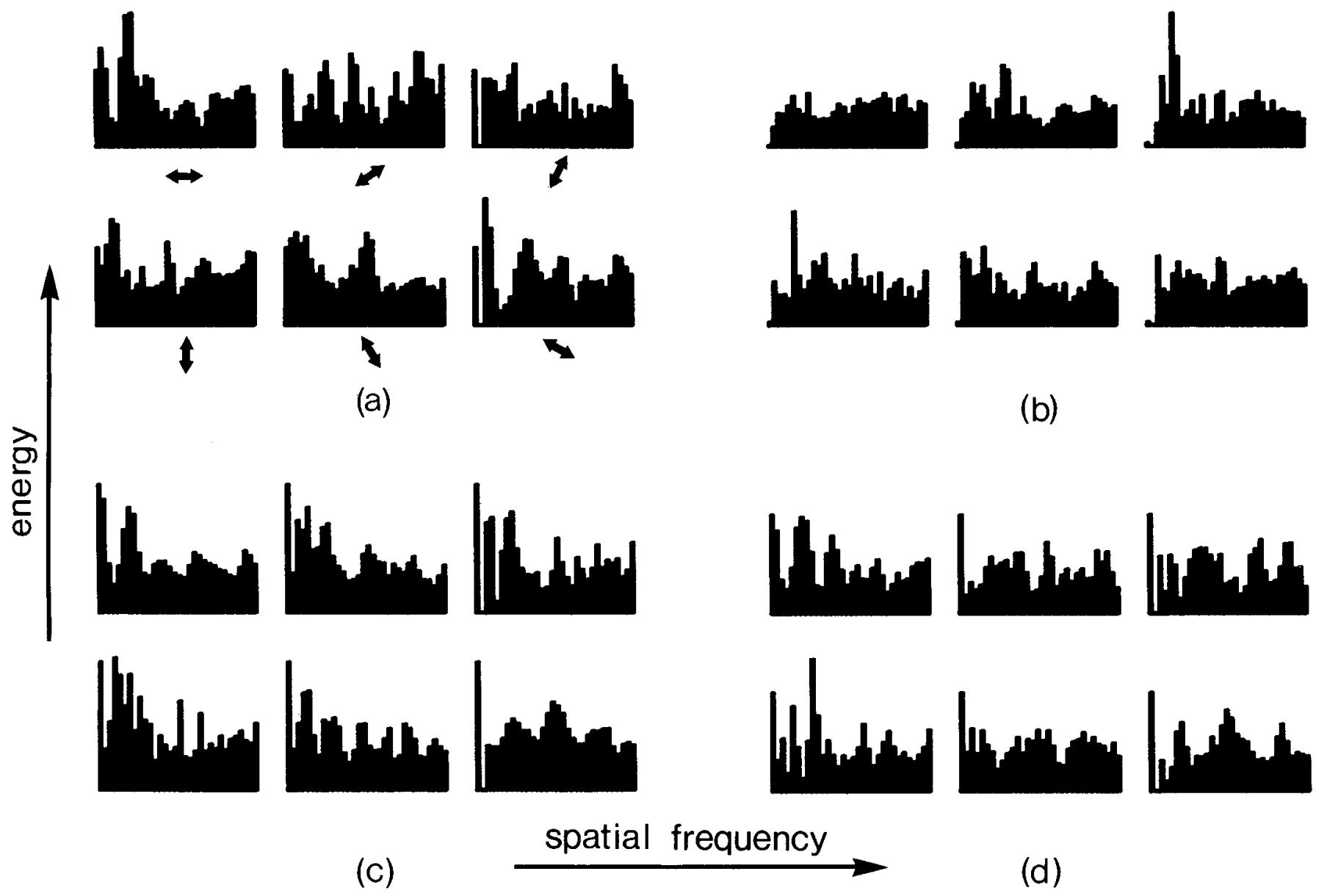

Figure 6. Fourier power spectra for the maps of direction selectivity for the same 4 animals shown in Figure 5.

this energy was a consequence of sampling with a quasiregular array of electrode penetrations, and (2) the effect of measurement error is to broaden any peaks in the spectrum. Our overall conclusion is that an ideal spectrum (obtained with an infinitely large number of completely accurate measurements) would have more sharply defined peaks and less energy above the sampling limit than those obtained in the present experiments.

3. In contrast to the orientation data, the power spectra obtained from the maps of direction preference (Fig. 6) showed no obvious structure and consisted of apparently uniform levels of noise at all frequencies.

\section{Interpolation}

In the Appcndix, we arguc that the shape of the orientation power spectrum justifies the use of sampling theory to interpolate orientation values for positions between those for which measurements were made. As a consequence, a detailed finegrain map of orientation could be reconstructed. Once Fourier transformation has been carried out, interpolation is a simple procedure, consisting of removing from the transform of the data the energy introduced as a result of sampling, i.e., by selling values at frequencies higher than the sampling limit to zero. An inverse Fourier transform is then applied to obtain the reconstructed map. While the arguments given in the Appendix suggest that this procedure ought to be accurate for the orientation maps, we thought it worthwhile to test it empirically. We deleted a single data point from the map, applied the interpolation procedure to the remaining data, and obtained an orientation value for the deleted point. This value was then compared with the one actually measured. This procedure was repeated in turn for every point of the map. Figure $8 a$, based on pooled data from 16 animals, shows that the interpolated orientation was most frequently correct, with the majority $(66 \%)$ of estimates being within $30^{\circ}$ of the correct value.

One might suppose that interpolation would be less accurate for points with a less than average number of neighbors (e.g., those near the edges of the maps), and when these were excluded from the analysis the accuracy of interpolation was improved (Fig. $8 b$ ), with about $79 \%$ of guesses being within $30^{\circ}$ of the correct value. Thus it is likely that when interpolation is carried out on the complete set of data values, the accuracy will be at least as good as this.

When the same test was carried out on the direction maps, estimates of preferred direction showed a greater than chance tendency to be correct (Fig. $8 \mathrm{c}$ ). Most of the correct guesses, however, came from 3 maps in which the sample spacing had been made much smaller than in the others. In the majority of the maps, directional interpolation appeared not to be an accurate procedure.

\section{Analysis of reconstructed orientation maps}

Figure 9 shows a reconstruction of the complete orientation map obtained in 1 animal, with the experimentally determined orientation values shown in red. Figure 10 shows the outlines of 
Figure 7. Overall distribution of (left) preferred orientation and (right) preferred direction obtained from a total of 2114 penetrations in 24 animals.
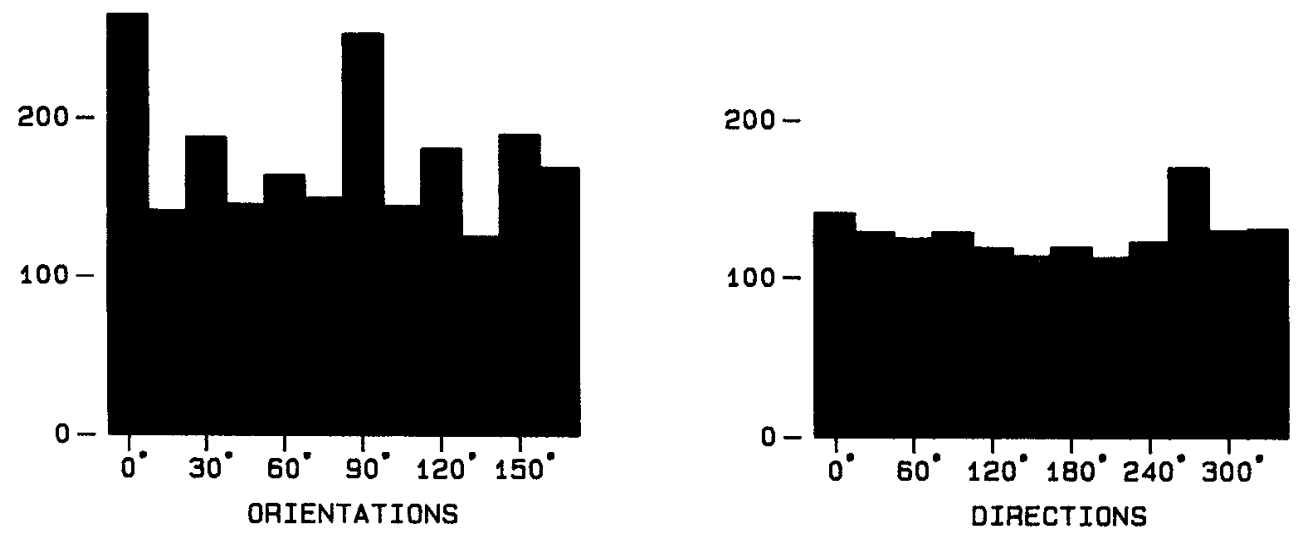

iso-orientation domains for $90^{\circ}$ orientation ranges in the same animal. In agreement with the results of deoxyglucose studies in areas 17 and 18 of the cat (Albus, 1979; Singer, 1981; Albus and Sieber, 1984), these domains show roughly parallel branching stripes, with an overall tendency to run in a direction, as deduced from the Fourier spectra, from anterolateral to posteromedial across the cortex. Examination of this and other completed maps showed that iso-orientation domains for orientation ranges $90^{\circ}$ wide each and individually $45^{\circ}$ apart (Fig. 10) had similar general properties - they had the same periodicity and ran in the same overall direction, but otherwise seemed to be independent. There was no obvious tendency for the different domain boundaries to avoid meeting, to avoid crossing, or to intersect at any particular angle. Probably because lines thrown down at random will intersect most frequently at $90^{\circ}$ (the frequency of intersections is proportional to the sine of the angle, and is thus maximal at $90^{\circ}$ ), intersections of domain boundaries at about $90^{\circ}$ seemed rather more common than cases where the boundaries touched or ran superimposed for short distances.

Figure 11 shows a color representation of the completed orientation map, using the color cycle red, orange, yellow, green, blue, and purple to represent the cycle of orientations. As there are 6 colors for an orientation range of $180^{\circ}$, each colored region in Figure 11 represents a $30^{\circ}$ range of orientation. Domains for such $30^{\circ}$ orientation ranges have a somewhat irregular shape, with boundaries that may be curved concavely or convexly, and giving rise to thin pointed extensions. Singularities-points in the map surrounded by a complete $180^{\circ}$ cycle of orientation domains - can easily be identified as points where the 6 different colors come together. These points also mark the crossings of domain boundaries referred to in the previous paragraph. They had an average spacing of about $750 \mu \mathrm{m}$. They were often apparent in the raw (uninterpolated) orientation maps. Figure 12 shows 4 examples: 2 where orientation changes clockwise for clockwise movements around the singularity, and 2 where the change is in an anticlockwise direction.

\section{Spatial layout of direction preference}

Before examining the maps of direction preference for evidence of structure, it is worth considering how structure and continuity in the map of direction preference must be constrained by the properties of the orientation map as described above. Given that the orientation map is continuous, with the exception of a number of $180^{\circ}$ singularities, and that direction preference is always orthogonal to orientation preference in one of 2 ways, the map of dircction preference cannot be continuous. Figure 13 shows that, in making a closed circuit around a single $180^{\circ}$ singularity, orientation changes (by definition) continuously through $180^{\circ}$. Direction preference, however, cannot similarly rotate without being forced to undergo at least one (or an odd number) of $180^{\circ}$ reversals. It is easy to show by construction (Fig. 13) that where there are orientation singularities, the map of direction preference must contain lines across which direction preference reverses, orientation preference remaining the same. Each orientation singularity must give rise to at least one such line, and each line must begin and end in a $180^{\circ}$ singularity. One conclusion to be drawn from this is that if singularities exist, reversals in the preferred direction must necessarily be frequently encountered in regions where the orientation preference changes smoothly. An electrode moving laterally through the cortex would be expected to encounter such reversals with roughly the same frequency as that of the spacing of singularitics.

Examination of our maps of direction preference provide a
Figure 8. a, A histogram of the differences between the interpolated orientation and that actually measured for 1627 points in 16 maps. $b$, Same as $a$, but excluding points with a relatively low density of neighbors. $c$, Differences between the interpolated direction preference and those actually measured for 1190 direction-selective points in 16 maps.
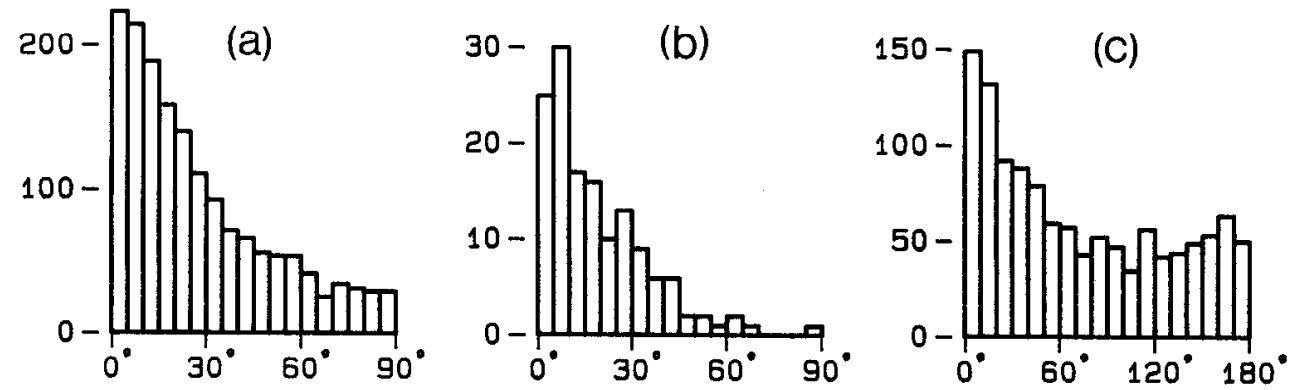

Predicted - measured Orientation 


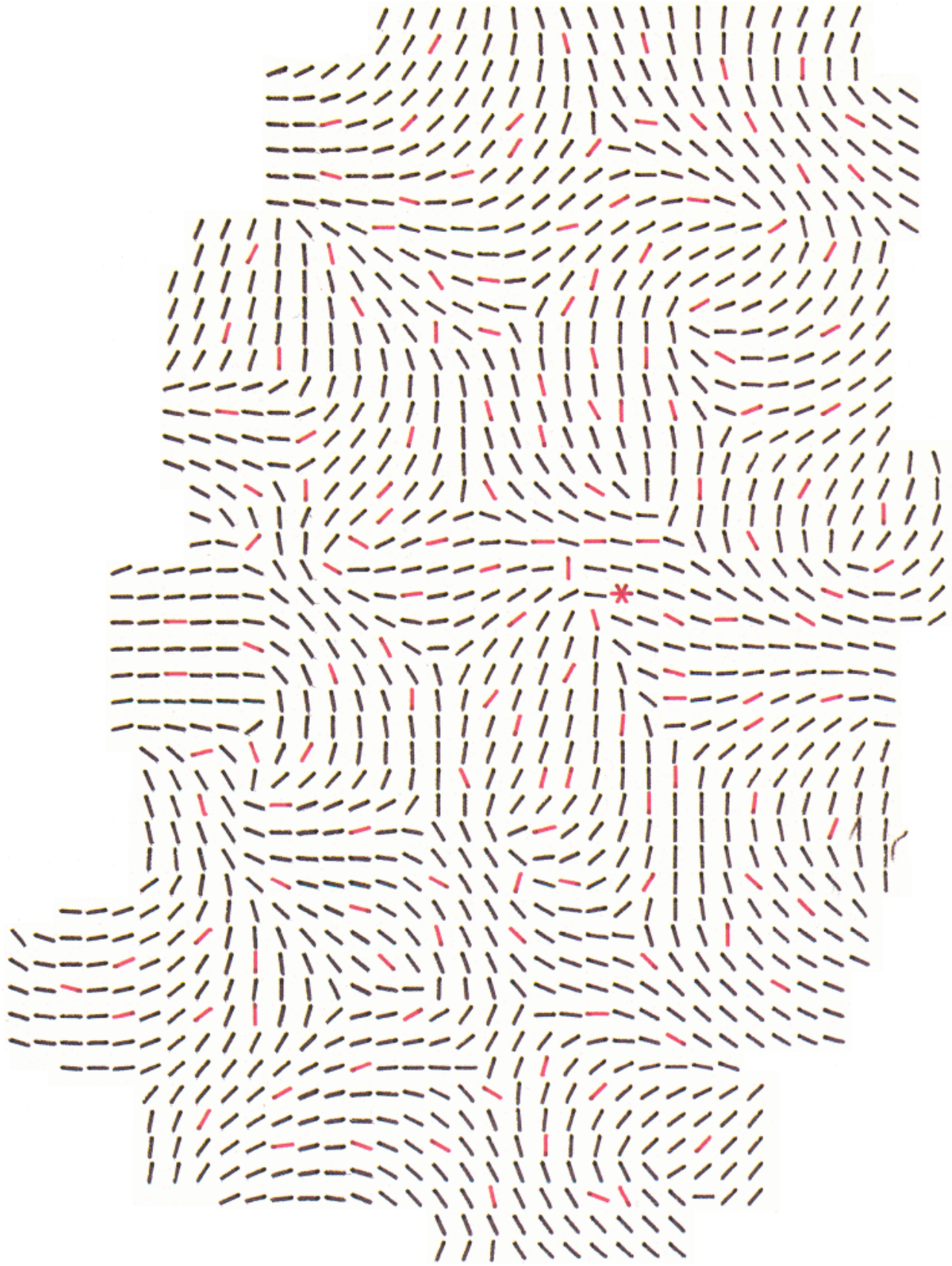

Figure 9. Reconstruction of the complete orientation map for a roughly $2.5 \times 3.5 \mathrm{~mm}$ area of cortex. Experimentally determined orientation values are shown in red. Asterisk marks a nonoriented response. Scale bar, $1 \mathrm{~mm}$; anterior is at bottom and medial is to the right. number of examples of regions containing a singularity, with a line of direction discontinuity extending outwards from it (Fig. $14, b, c)$. Elsewhere it was possible to find areas within which orientation preference varied smoothly, divided into regions of opposed direction preference (Fig. 14a). In a few cases, lines of direction discontinuity could be reconstructed over an entire map of direction preference (Fig. 15). It was helpful, when doing this, to use the map of interpolated orientation preference to decide whether 2 points with direction selectivities that were, e.g., $90^{\circ}$ apart were likely to be divided by a discontinuity or not. This would depend on whether orientation changed clockwise or anticlockwise between the 2 points. Such reconstructions showed that the majority of singularities gave rise to a single line of discontinuity; these lines were usually curved and normally failed to connect singularities by the shortest possible routes, although they usually connected singularities that were close together, if not nearest neighbors. There was no obvious tendency for the lines to run across the cortex in any particular direction or to run orthogonal to the boundaries of the isoorientation domains. Nor was there any obvious preferred orientation at which the direction reversals would occur, the orientation often varying along the length of such lines.

\section{Discussion}

The map of orientation

Our conclusions about the map of orientation preferences in area 18 can be summarized as follows: (1) Orientation changes continuously nearly everywhere, except for a number of point singularities, each of which is surrounded by a $180^{\circ}$ cycle of orientation values; (2) orientation changes periodically, a com- 


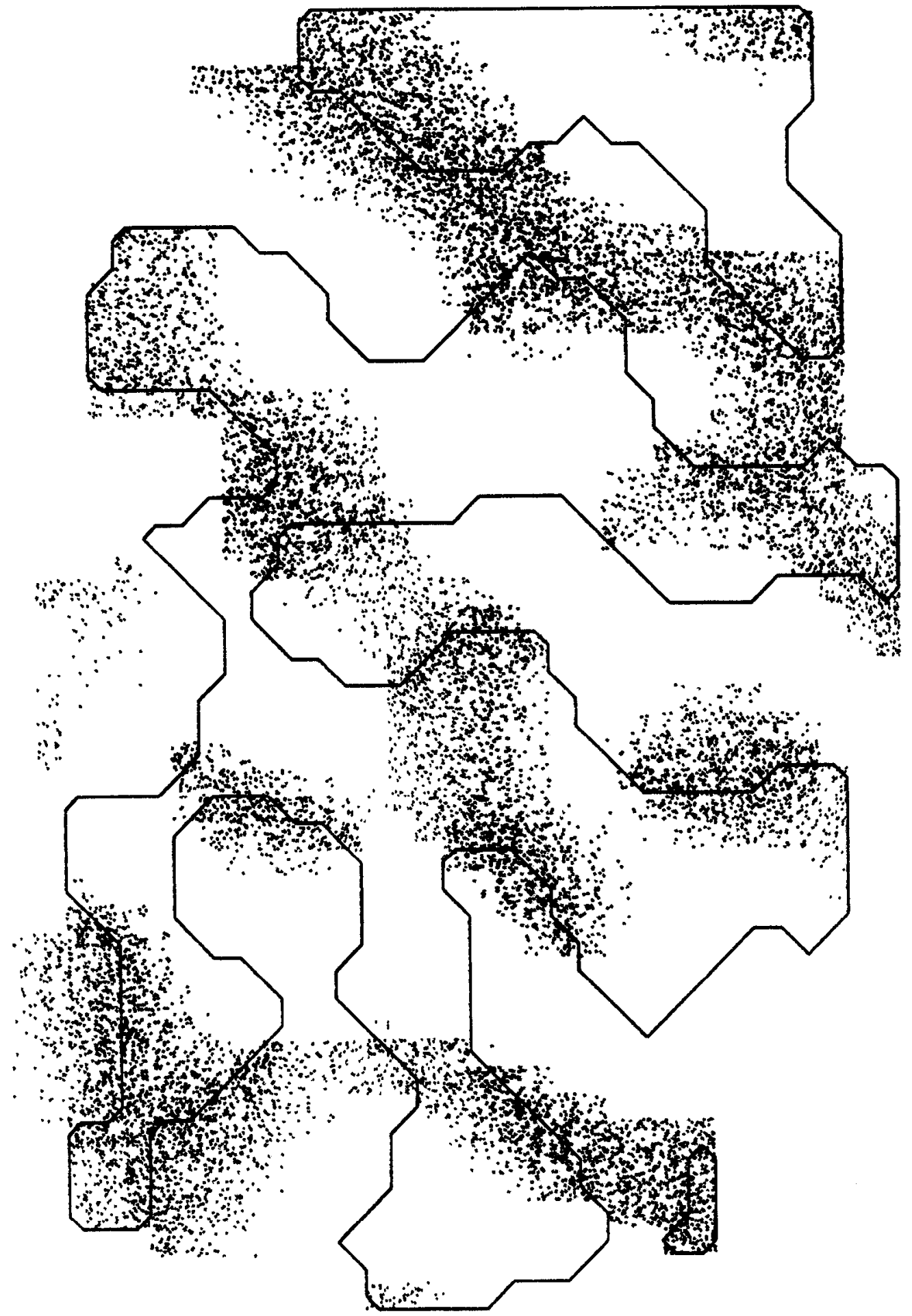

Figure 10. Iso-orientation domains for $90^{\circ}$ wide ranges in the map shown in Figure 9; scale of the maps is the same. Solid black line surrounds domains centered on horizontal and vertical, i.e., ranges from $-45^{\circ}$ to $+45^{\circ}$ and $45^{\circ}-135^{\circ}$, respectively. Dotted regions show domains for the range $0^{\circ}-90^{\circ}$. Note the apparent independence of the 2 sets of domains. Singularities occur where the 2 types of domain boundaries intersect.

plete $180^{\circ}$ cycle occurring on average every $1.25 \mathrm{~mm}$; (3) isoorientation domains have an overall tendency to run from lateral to medial at an angle of about $30^{\circ}$ from the M-L axis; (4) isoorientation domains for orientation ranges that are each $90^{\circ}$ wide and individually $45^{\circ}$ apart appear to be independent, except for a tendency to run in a similar direction and to have similar center-to-center spacing.
The accuracy of this description depends to a large extent on the accuracy of the interpolation procedure used to estimate orientation preference in regions where no physiological recordings were made. While we believe that the quantitative arguments used to justify the procedure are adequately rigorous, we have been unable to estimate on mathematical grounds the probable accuracy of interpolation. The empirical test ádopted 




Figure 11. Color representation of a completed orientation map, using the color cycle red, orange, yellow, green, blue, and violet to represent the cycle of orientations. Each of the 6 colors represents an orientation range of $30^{\circ}$, centered on horizontal (red), $30^{\circ}$ (orange), $60^{\circ}$ (yellow), vertical (green), $120^{\circ}$ (blue), and $150^{\circ}$ (violet).

here has the virtue of being foolproof, but it can only place an upper limit-about $\pm 30^{\circ}$ - on the accuracy of the interpolated orientation values. This is probably rather less accurate than reasonably careful measurements based on auditory judgements of response could achieve. There are, however, reasons for thinking that the interpolation procedure may be more accurate than this estimate suggests. Consider the ideal case of sampling from a band-limited function, with sample points regularly spaced at the sampling limit. In such a case the information present in the value measured at each point would be minimally redundant, and would not necessarily carry information about the values of surrounding points. Deleting one such value and at- 


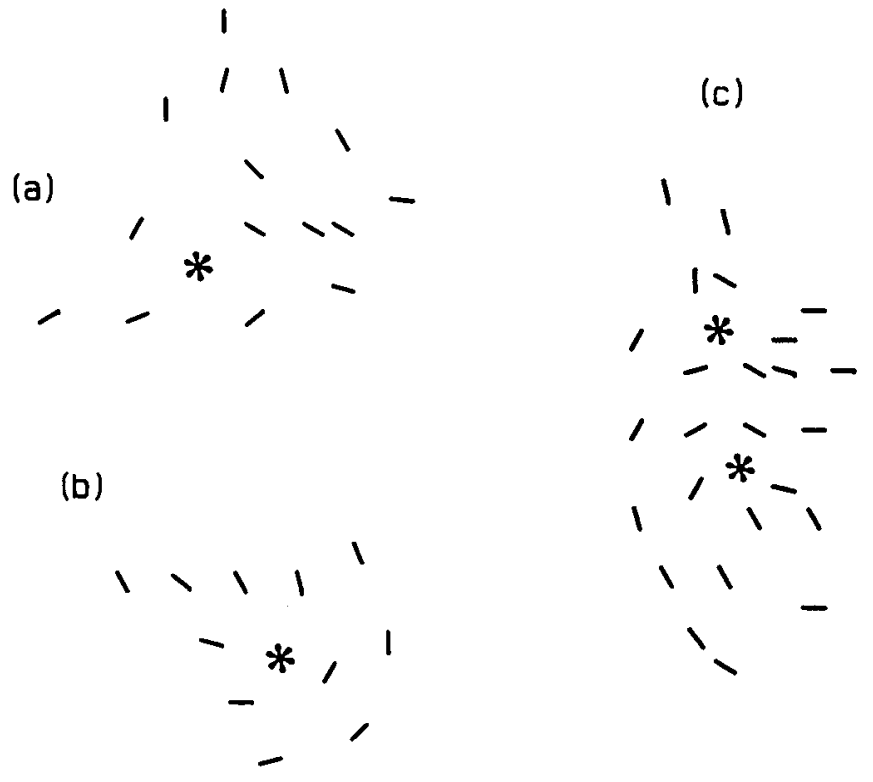

Figure 12. Examples of $180^{\circ}$ singularities occurring in the raw orientation maps. Stars in this and subsequent diagrams mark the approximate center of each singularity. Note that in making a single circuit in a given direction (e.g., clockwise) around a singularity, the orientations rotate either clockwise or anticlockwise through $180^{\circ}$. a, c (upper half), Rotation is anticlockwise. $b, c$ (lower half), Rotation is clockwise.

tempting to estimate it by interpolation, as we have done, would not be accurate, although the procedure would be perfectly accurate when performed using the complete set of sampled values. In the same way, our interpolations are likely to be significantly more accurate when performed using the complete set of data values.

To what extent does the interpretation of orientation domain structure depend on the accuracy of the interpolations? If these are correct to within $\pm 30^{\circ}$, the positions of the domain boundaries must be similarly uncertain, by a distance equal to onesixth (30/180) of the period of the domains. It is unlikely that any manipulation of the positions of domain boundaries by this amount would produce a radically different type of organization from the one proposed here.

Our results conform to an arrangement proposed earlier (Swindale, 1982) on the basis of a computer model of how orientation columns might develop. The assumptions of the model were that orientation selectivity was not intrinsically determined for any one cell or group of cells, but was initially only slight and randomly biased. Following this, orientation selectivity was assumed to increase, subject only to the constraint that cells less than about $250 \mu \mathrm{m}$ apart would tend to have similar preferences, while cells further apart (e.g., from 250 to $500 \mu \mathrm{m}$ ) would tend to develop orthogonal preferences. This resulted in a pattern of orientations with a power spectrum that was sharply peaked at a particular distance from the origin, in agreement with our present experimental results. Two other features of the pattern of orientations produced by the model agree with our experimental data. One is the apparent independence of the patterns of iso-orientation domains for orientation ranges $90^{\circ}$ apart (Fig. 10), while the other is the presence of halfrotation $\left(180^{\circ}\right)$ singularities spaced with approximately twice the frequency of the iso-orientation domains. An electrode traveling laterally through the cortex and passing through such a singularity would record a sudden $90^{\circ}$ shift in orientation pref-

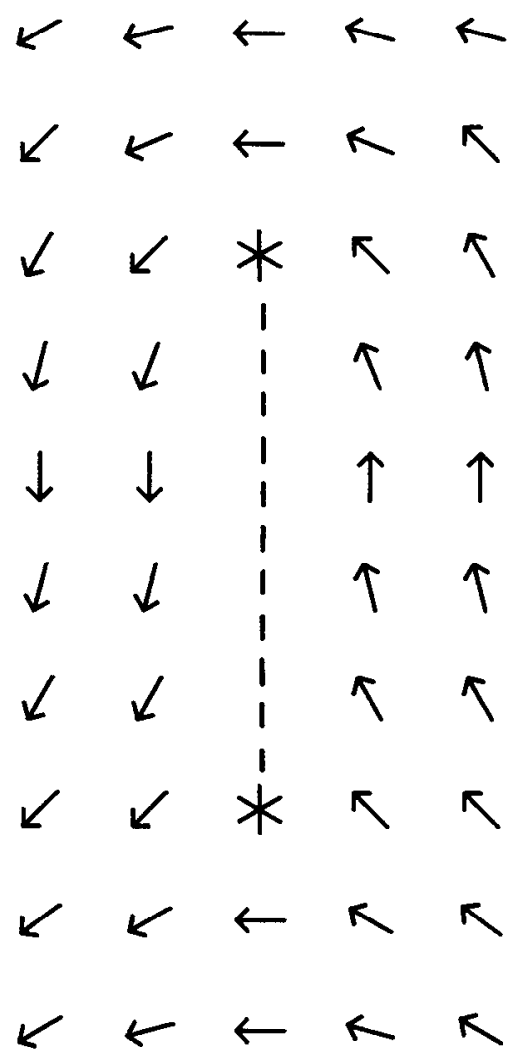

Figure 13. Diagram showing 2 hypothetical orientation singularities, around which directions are arranged as continuously as possible. There must inevitably be a line across which direction selectivity reverses, joining the 2 singularities.

erence. These shifts are observed in area 17 of the cat (Albus, 1985 ) and in the monkey visual cortex (IIubel and Wiesel, 1974; Livingstone and Hubel, 1984), suggesting that the properties of the map of orientation preference are similar in both the cat and the monkey. Albus's $(1975,1985)$ data from area 17 of the cat, obtained from recording sites spaced $100 \mu \mathrm{m}$ apart along single long penetrations, are also consistent with the type of organization proposed here for area 18.

Our findings from cat area 18 are similar to the recent results of Blasdel and Salama (1986), who used voltage-sensitive dyes to map the layout of orientation preference in area 17 of the monkey. (For example, compare our Fig. 11 with figs. 5a and 6a of Blasdel and Salama, 1986.) Although not mentioned by the authors, the voltage-sensitive dye maps clearly contain $180^{\circ}$ singularities, identifiable as points where the sharp extensions of a single set of differently colored domains meet. The spacing of these singularities in relation to the overall periodicity of the domains appears to be about the same as that observed herc in the cat. Periodic variations in orientation gradient also exist in our maps, as they do in the monkey (Blasdel and Salama, 1986) and also in the computer model. However, our maps are probably not accurate enough to allow regions of increased orientation gradient to be distinguished from discrete linear fractures in the orientation map, as Blasdel and Salama claim exist for the monkey.

Evidence for the arrangement suggested by Braitenberg and Braitenberg (1979), in which there are periodically spaced $360^{\circ}$ singularities around which orientations are arranged, either radially or tangentially, was not evident in our data, either in the 


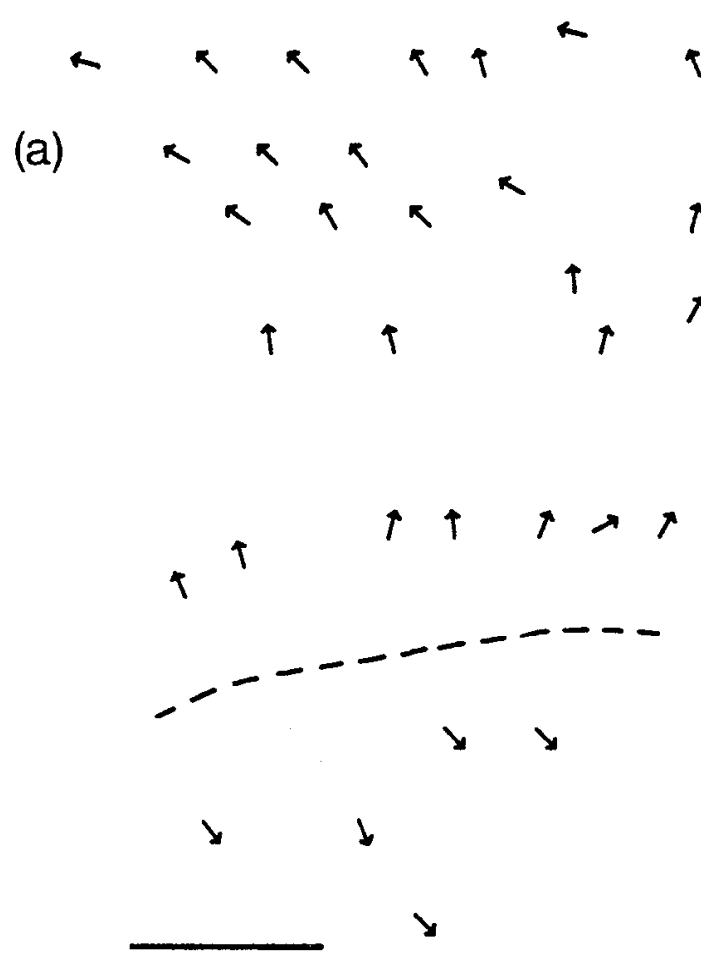

raw or interpolated orientation maps. Albus (1985) has also argued that orientation data from cat area 17 (Albus, 1975, 1985) are inconsistent with this type of model.

\section{Map of direction preference}

Our conclusions about the map of direction preference in area 18 are that (1) there is a strong tendency for neighboring points to have the same direction preference; (2) there are regions within which direction preference changes continuously, incompletely bounded by lines across which direction preference reverses; (3) these lines begin and end in orientation singularities; and (4) they do not necessarily join together singularities that are nearest neighbors, or take the shortest routes between those singularities they do connect.

These conclusions are consistent with those of others who have studied the organization of direction preference in cat area 17 (Payne et al., 1981; Tolhurst et al., 1981). Both groups show, in a manner similar to that used here for area 18 , a tendency for the direction preferences of units less than $350 \mu \mathrm{m}$ apart to be similar. Payne et al. (1981) also present evidence of regions of continuous change, both in orientation and direction preference, interrupted by $180^{\circ}$ reversals in the preferred direction. These direction reversals occur on average every 500-600 $\mu \mathrm{m}$ of lateral movement throughout the cortex. This is similar to the distance between the lines of discontinuity in direction preference observed in our maps, and is good independent evidence that the organization proposed here for direction sclectivity actually prevails in the visual cortex.

\section{Appendix}

We consider 2 factors that affect the shape of the power spectrum: experimental error in the determination of orientation preference, and the effects of sampling with a quasiregular array

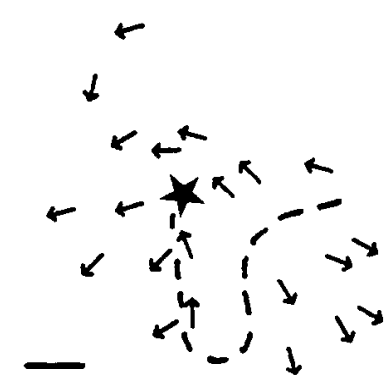

C)

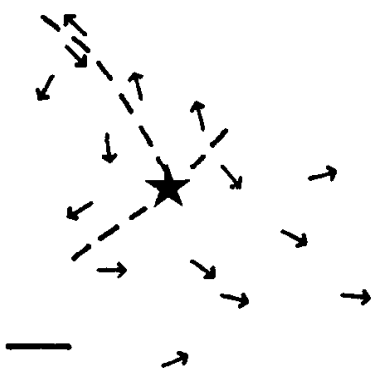

Figure 14. Regions from the raw maps of direction preferences showing $(a)$ single line of direction discontinuity dividing 2 regions of opposed direction preference; $(b)$ curved line of discontinuity originating in a singularity; and (c) evidence that more than 1 line of discontinuity may originate from a singularity. Scale bars, $200 \mu \mathrm{m}$.

of electrode penetrations. We show that the effect of measurement error is to broaden any peaks present in the spectrum, while the effect of sampling is to introduce unstructured noise into the spectrum at frequencies above the sampling limit. The

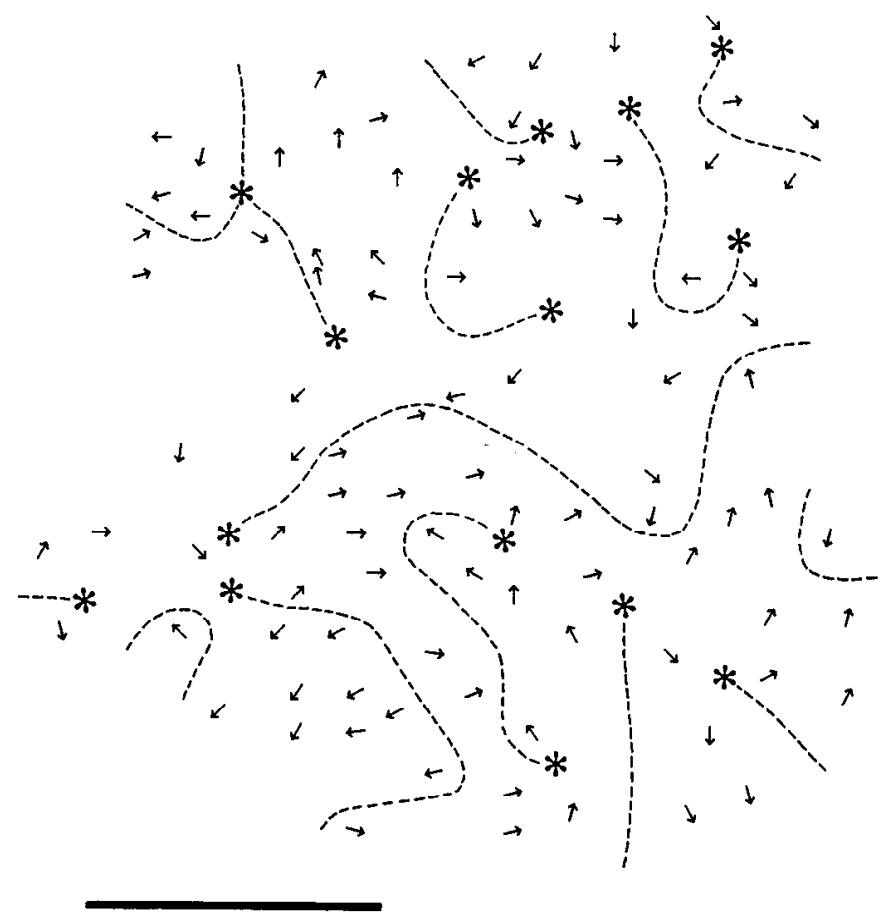

$1 \mathrm{~mm}$

Figure 15. Reconstruction showing the raw map of direction selectivity obtained in one animal (arrows indicate preferred direction of movement); the distribution of orientation singularities in the map (asterisks) determined by orientation interpolation; and the probable layout of the lines of direction discontinuity joining the singularities (dashed lines). 
ideal spectrum, obtained with an infinitely large number of completely accurate measurements, would necessarily have more sharply defined peaks and less energy present above the sampling limit than those obtained in the present experiments. Our arguments are based on standard sampling theory, as outlined, for example, in Bracewell (1965).

\section{Effect of measurement error}

It is likely that the experimental measurements of preferred orientation are not always correct, but deviate randomly from the value that might be obtained by a more thorough measurement or a sample from a larger number of cells within a single vertical penetration. The size of this deviation is not known, but we estimate that it might be as large as $\pm 15^{\circ}$. The effect of this on the spectrum is as follows: neglecting sampling effects (which are discussed in the following section, and can be incorporated into the present argument without affecting it) and using the relation $\mathbf{z}=\exp (2 i \theta)$, the measured value of $\mathbf{z}$ is $\mathbf{z}^{\prime}=$ $\exp \left[2 i\left(\theta+\theta_{e}\right)\right]$, where $\theta$ is the actual value of orientation and $\theta_{e}$ is the error. This can be written as $\mathbf{z}^{\prime}=\mathbf{z} \mathbf{z}_{e}$, where $\mathbf{z}_{e}=\exp \left(2 i \theta_{e}\right)$, and has the transform $\mathbf{Z}^{\prime}=\mathbf{Z} * \mathbf{Z}_{e}^{\prime}$, where $\mathbf{Z}$ is the Fourier transform of $\mathbf{z}$ and $*$ denotes convolution. If measurement error is zero, $z=\exp (0)$ is unity everywhere, and its transform is a delta function at the origin. Convolution with this preserves the original spectrum everywhere, as would be expected. If instead $\mathbf{z}$ is normally distributed around a mean of 0 , its transform will be an approximately Gaussian function centered on the origin, with a lateral spread proportional to the standard deviation of $\mathbf{z}_{e}$; convolution with this will broaden any peaks in the spectrum, rather than adding uniform noise at all frequencies (as one might otherwise have supposed). Given that some degree of measurement error is certain to be present, the actual peaks in the spectra shown here must necessarily be narrower than the measured ones.

\section{Effects of discrete sampling on the spectrum}

If orientation, measurcd as a function of position $\mathbf{r}$ on the cortical surface, is the function $\mathbf{z}^{\prime}(\mathbf{r})$, and is sampled with the function $s(\mathbf{r})$, where $s$ has the value 1 at a sample point and is zero elsewhere, the resulting signal is $\mathbf{z}^{\prime \prime}=s \mathbf{z}^{\prime}$. This has the transform $\mathbf{Z}^{\prime \prime}=\mathbf{S} * \mathbf{Z}^{\prime}$, where $\mathbf{S}$ is the transform of $s$. The problem is to deduce the properties of $\mathbf{Z}$, given information only about $Z^{\prime \prime}$. If sampling is done with a regularly spaced array of points (e.g., a square array), the transform of this sampling function is also a square array of points. The effect of convolving the original transform with such a function is to cause it to be replicated periodically in Fourier space. Unless the original signal is known to be band-limited (i.e., contained within a region that lies within the peaks of the sampling spectrum), which cannot be assumed to be the case here, it is impossible to deduce with certainty the form of the original spectrum. However, as was recently pointed out by Yellott (1982), this problem does not arise when the sampling function is a quasiregular array, as here. In this case the transform of the sampling function is sharply peaked at the origin, the peak being surrounded by a roughly circular clear zone, where the value of the function is zero, the radius of this clear zone being inversely proportional to the spacing of the samples. Surrounding this is a further annulus of (effectively) unstructured noise. Any sharp peaks in the original data spectrum will be preserved in their original positions by convolution with such a function; instead of being replicated at frequencies above the sampling limit, such peaks will be smeared out and contribute to a steady noise background. Energy peaks above the sampling limit will likewise be preserved and contribute to noise levels below this limit.

From these arguments, one can deduce that (1) the peaks present in the spectra shown in Figure 5 must be present in the original spectra at the same frequency, although some degree of broadening is produced as a result of measurement error; (2) the energy in the spectra that is above the sampling limit (determined to be the frequency with a period twice the average spacing between the sample points) is at least in part due to scattering of the energy present below this frequency, as a result of sampling with a quasiregular array; (3) because the energy levels on either side of the peak (i.e., close to zero frequency and just below the sampling limit) are low, there must be little energy present in the original signal above the sampling limit, since this would appear as "background" noise at all frequencies below this limit. The corollary of this argument is that if most of the energy (and thus information) in the original signal is confined to frequencies below the sampling limit, sampling theory can be used to interpolate orientation values for positions between those where measurements were made, allowing a finegrain reconstruction of the original orientation map. Finally, (4) these arguments for the validity of interpolation do not apply with any force to the maps of direction preference, since the power spectrum is flat. It is possible that most of the original energy in the spectrum is below the sampling limit, which would allow interpolation, but one cannot be certain that this is the case.

\section{References}

Albus, K. (1975) A quantitative study of the projection area of the central and paracentral visual field in area 17 of the cat. II. The spatial organisation of the orientation domain. Exp. Brain Res. 24: 181-202.

Albus, K. (1979) ${ }^{14} \mathrm{C}$-deoxyglucose mapping of orientation subunits in the cat's visual cortical areas. Exp. Brain Res. 37: 609-613.

Albus, K. (1985) A microelectrode study of the spatial arrangement of iso-orientation bands in the cat's striate cortex. In Models of the Visual Cortex, D. Rose and V. Dobson, eds., pp. 485-491, Wiley, New York.

Albus, K., and B. Sieber (1984) On the spatial arrangement of isoorientation bands in the cat's visual cortical areas 17 and 18 : $\mathrm{A}{ }^{14} \mathrm{C}$ deoxyglucose study. Exp. Brain Res. 56: 384-388.

Batschelet, E. (1981) Circular Statistics in Biology, Academic, New York.

Bergland, G. D. (1969) A guided tour of the fast Fourier transform. Spectrum 6: 41-52.

Blasdel, G. G., and G. Salama (1986) Voltage-sensitive dyes reveal a modular organisation in monkey striate cortex. Nature 321: 579-585.

Bracewell, R. (1965) The Fourier Transform and its Applications, McGraw-Hill, New York.

Braitenberg, V., and C. Braitenberg (1979) Geometry of orientation columns in the visual cortex. Biol. Cybern. 33: 179-186.

Brigham, E. O. (1974) The Fast Fourier Transform, Prentice-Hall, Englewood Cliffs, New Jersey.

Cynader, M. S., N. V. Swindale, and J. A. Matsubara (1987) Functional topography in cat area 18. J. Neurosci. 7: 1401-1413.

Hubel, D. H., and T. N. Wiesel (1962) Receptive fields, binocular interaction and functional architecture in the cat's visual cortex. $\mathrm{J}$. Physiol. (Lond.) 160: 106-154.

Hubel, D. H., and T. N. Wiesel (1963) Shape and arrangement of columns in cat's striate cortex. J. Physiol. (Lond.) 165: 559-568.

Hubel, D. H., and T. N. Wiesel (1968) Receptive fields and functional architecture of monkey striate cortex. J. Physiol. (Lond.) 195: 215243.

Hubel, D. H., and T. N. Wiesel (1974) Sequence regularity and ge- 
ometry of orientation columns in the monkey striate cortex. J. Comp. Neurol. 158: 267-294.

Hubel, D. H., T. N. Wiesel, and M. P. Stryker (1978) Anatomical demonstration of orientation columns in macaque monkey. J. Comp. Neurol. 177: 361-380.

Humphrey, A. L., and T. T. Norton (1980) Topographic organisation of the orientation column system in the striate cortex of the tree shrew (Tupaia glis). I. Microelectrode recording. J. Comp. Neurol. 192: 531547.

Humphrey, A. L., L. C. Skeen, and T. T. Norton (1980) Topographic organisation of the orientation column system in the striate cortex of the tree shrew (Tupaia glis). II. Deoxyglucose mapping. J. Comp. Neurol. 192: 549-566.

Kennedy, H., and G. A. Orban (1979) Preference for horizontal or vertical orientation in cat visual cortical neurons. J. Physiol. (Lond.) 296: 61P-62P.

Leventhal, A. G., and H. V. B. Hirsch (1977) Effects of early visual experience upon orientation sensitivity and binocularity of neurons in visual cortex of cats. Proc. Natl. Acad. Sci. USA 74: 1272-1276.

I ivingstone, M. S., and D. H. Hubel (1984) Anatomy and physiology of a color system in the primate visual cortex. J. Neurosci. 4: 309356.

Matsubara, J. A., M. Cynader, and N. V. Swindale (1987) Anatomical properties and physiological correlates of the intrinsic connections in cat area 18. J. Neurosci. 7: 1428-1446.

Payne, B. R., N. Berman, and E. H. Murphy (1981) Organisation of direction preferences in cat visual cortex. Brain Res. 211: 445-450.

Singer, W. (1981) Topographic organisation of orientation columns in the cat visual cortex. Exp. Brain Res. 44: 431-436.

Swindale, N. V. (1982) A model for the formation of orientation columns. Proc. R. Soc. Lond. [Biol.] 215: 211-230.

Swindale, N. V. (1985) Iso-orientation domains and their relationship with cytochrome oxidase patches. In Models of the Visual Cortex, D. Rose and V. Dobson, eds., pp. 452-461, Wiley, New York.

Tolhurst, D. J., A. F. Dean, and I. D. Thompson (1981) Preferred direction of movement as an element in the organisation of cat visual cortex. Exp. Brain Res. 44: 340-342.

Yellott, J. I. (1982) Spectral analysis of spatial sampling by photoreceptors: Topological disorder prevents aliasing. Vision Res. 22: 12051210 . 